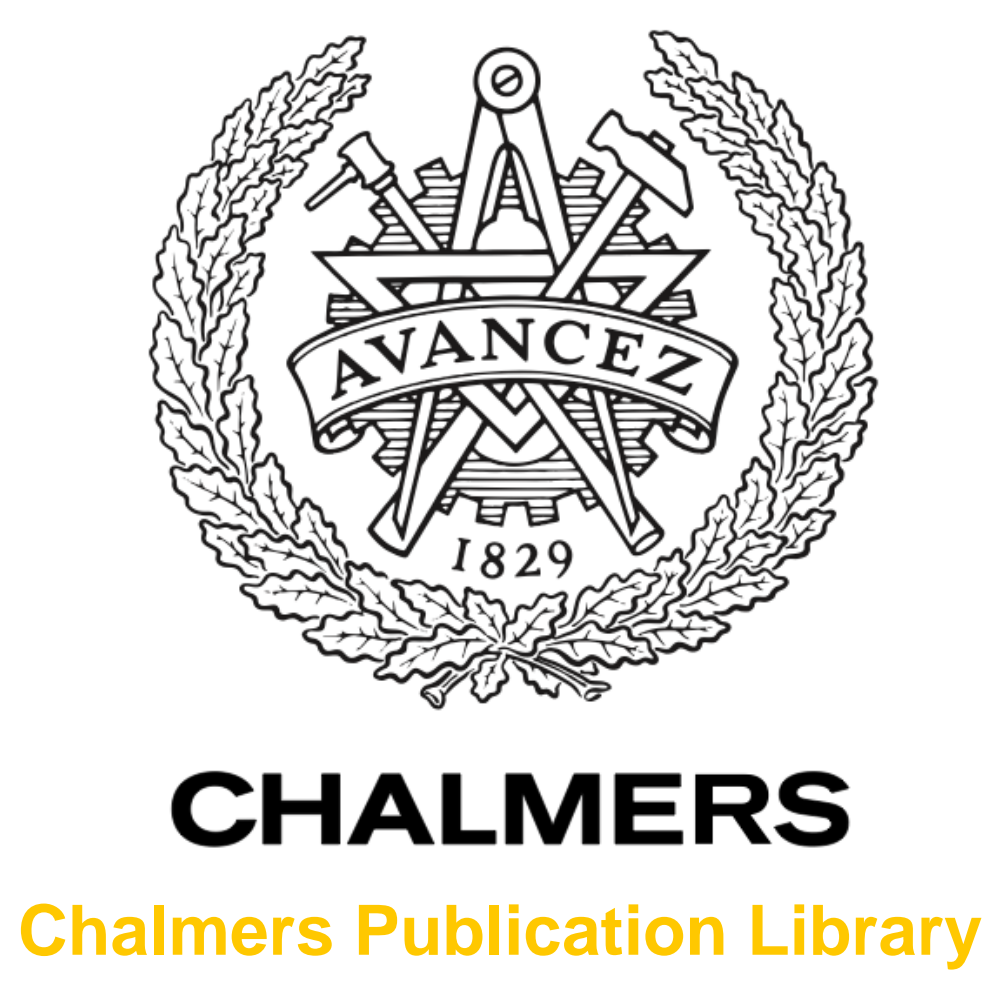

Noise-based antenna terms for active receiving arrays

This document has been downloaded from Chalmers Publication Library (CPL). It is the author's version of a work that was accepted for publication in:

IEEE International Symposium on Antennas and Propagation, Chicago, USA, July 8-14, 2012 (ISSN: 1522-3965)

Citation for the published paper:

Warnick, K. ; Ivashina, M. ; Maaskant, R. (2012) "Noise-based antenna terms for active receiving arrays". IEEE International Symposium on Antennas and Propagation, Chicago, USA, July 8-14, 2012

http://dx.doi.org/10.1109/APS.2012.6348696

Downloaded from: http://publications.lib.chalmers.se/publication/169402

Notice: Changes introduced as a result of publishing processes such as copy-editing and formatting may not be reflected in this document. For a definitive version of this work, please refer to the published source. Please note that access to the published version might require a subscription.

Chalmers Publication Library (CPL) offers the possibility of retrieving research publications produced at Chalmers University of Technology. It covers all types of publications: articles, dissertations, licentiate theses, masters theses, conference papers, reports etc. Since 2006 it is the official tool for Chalmers official publication statistics. To ensure that Chalmers research results are disseminated as widely as possible, an Open Access Policy has been adopted.

The CPL service is administrated and maintained by Chalmers Library. 


\title{
Noise-Based Antenna Terms for Active Receiving Arrays
}

\author{
Karl F. Warnick*, Marianna Ivashina ${ }^{\dagger}$, Rob Maaskant ${ }^{\ddagger}$, and Bert Woestenburg ${ }^{\S}$ \\ *Electrical \& Computer Engineering Dept., Brigham Young University, Provo, Utah, USA, Email: warnick@ee.byu.edu \\ ${ }^{\dagger}$ Department of Earth and Space Sciences, Chalmers University of Technology, Gothenburg, Sweden \\ $¥$ Department of Signals and Systems, Chalmers University of Technology, Gothenburg, Sweden \\ $\S$ Netherlands Institute for Radio Astronomy, Dwingeloo, The Netherlands
}

\begin{abstract}
For active receiving arrays with complex receiver chains, nonreciprocal components in the beamforming network, or digitally sampled and processed output signals, existing transmit-based antenna terms such as gain and radiation efficiency cannot be directly applied. Using the reciprocity principle to obtain an equivalence between the total power radiated by a transmitting antenna and the noise power at the output of a receiving antenna, a new set of more general, receiverspecific antenna terms have been obtained. For passive, reciprocal antennas, the new definitions are rigorously equivalent to existing definitions. The terms have received the IEEE Antenna Definitions Working Group's support for inclusion pending final approval by the Antenna Standards Committee in the next update of the IEEE standard for antenna terms.
\end{abstract}

\section{INTRODUCTION}

Active multi-antenna systems and antenna arrays are of great interest currently for applications such as high-sensitivity astronomical aperture phased arrays and phased array feeds, multiple input multiple output (MIMO) communications systems, digitally beamformed arrays, steered beam antennas for passive remote sensing, and arrays for mobile, airborne, and maritime satellite communications. The standard definitions for gain, radiation efficiency, antenna efficiency, and noise temperature are directly applicable only to receiving antennas that can be operated as transmitters. When the antenna system is complex and includes active and nonreciprocal components, or the beam output is realized in digital signal processing, more general definitions for these figures of merit are needed.

There are several available figures of merit that can be applied to arbitrarily complex, active receivers, including receiving pattern directivity, solid-beam efficiency, and embedded element efficiency [1]. In the array signal processing literature, array gain is the SNR at the output of a sensor array relative to the SNR of a single sensor. Array gain can be taken relative to the standard directivity to produce an array efficiency figure of merit [2]. With the exception of directivity, these existing figures of merit are not used uniformly throughout the antenna community or have not been incorporated into the IEEE standard for antenna terms.

More recently, it was found that some of these nonstandard figures of merit are connected in a fundamental way to conventional notions of antenna gain and efficiency [3]. This result was exploited to define efficiencies and system noise temperature for active, nonreciprocal, and digitally beamformed array antennas $[4,5]$. An early version of the new antenna parameters was reported along with theoretical justification in [6]. The resulting definitions, including refinements and clarifications that arose during discussions with the IEEE Antenna Standards Working Group, have been formulated into the concise set of proposed antenna terms presented below.

\section{NEW ANTENnA TERMS}

Following is a list of the new antenna terms and definitions. Text that already exists in the current IEEE Standard Definitions of Terms for Antennas [7] is italicized.

isotropic noise response. For a receiving active array antenna, the noise power at the output of a formed beam with a noiseless receiver when in an environment with brightness temperature distribution that is independent of direction and in thermal equilibrium with the antenna.

active antenna available gain. For a receiving active array antenna, the ratio of the isotropic noise response to the available power at the terminals of any passive antenna over the same bandwidth and in the same isotropic noise environment.

active antenna available power. For a receiving active array antenna, the power at the output of a formed beam divided by the active antenna available gain.

2.251 noise temperature of an antenna. The temperature of a resistor having an available thermal noise power per unit bandwidth equal to that at the antenna output at a specified frequency. For an active antenna, the temperature of an isotropic thermal noise environment such that the isotropic noise response is equal to the noise power at the antenna output per unit bandwidth at a specified frequency.

2.115 effective area (of an antenna) (in a given direction). In a given direction, the ratio of the available power at the terminals of a receiving antenna to the power flux density of a plane wave incident on the antenna from that direction, the wave being polarization matched to the antenna. See: polarization match.

NOTES

1-If the direction is not specified, the direction of maximum radiation intensity is implied.

2-The effective area of an antenna in a given direction is equal to the square of the operating wavelength times its gain in that direction divided by $4 \pi$.

3 - For an active antenna, available power is the active antenna available power.

Receiving efficiency. For a receiving active array antenna, the ratio of the isotropic noise response with noiseless antenna to the isotropic noise response, per unit bandwidth and at a specified frequency.

Noise matching efficiency. For a receiving active array antenna, the ratio of the noise power contributed by receiver electronics at the output of a formed beam, with receivers impedance matched to the array elements for minimum noise, to the actual receiver electronics noise power at the formed beam output, per unit bandwidth and at a specified frequency. 
The theoretical basis for the new definitions is that whereas transmit antenna parameters are normalized by total radiated power or input power, for receivers the appropriate normalization factor is the noise power $P_{\mathrm{t} \text {,iso }}$ at the output of the antenna when the antenna is immersed in an environment with brightness temperature $T_{\text {iso }}$ that is equal in all directions, or an isotropic thermal noise environment. $P_{\mathrm{t} \text {,iso }}$ includes noise produced by antenna losses, with the antenna structures at the same temperature $T_{\mathrm{iso}}$ as the scene around the antenna, but does not include noise added by receiver electronics. The isotropic noise response can be measured directly by surrounding an antenna under test with hot and cold black bodies of known brightness temperature.

The isotropic noise response can be used to define the active antenna available gain $G_{\mathrm{rec}}^{\mathrm{av}}=P_{\mathrm{t} \text {,iso }} /\left(k_{\mathrm{b}} T_{\text {iso }} B\right)$, where $k_{\mathrm{b}}$ is Boltzmann's constant and $B$ is the noise equivalent system bandwidth. Alternately, the active antenna available gain can be defined as the isotropic noise power spectral density at the output relative to $k_{\mathrm{b}} T_{\text {iso }}$. The significance of the active antenna available gain is that it allows the active antenna available output power to be defined as $P_{\text {out }}^{\text {av }}=P_{\text {out }} / G_{\text {rec }}^{\text {av }}$ for any receiving antenna system, whether the antenna is passive, includes gain stages in one or more signal paths, or the output is formed in digital signal processing as a beam, image pixel, or a MIMO spatial code. As long as the system has an identifiable output signal, available output power is well defined. Loosely, the active antenna available power is the power at the antenna output port with receiver gain removed, and reduces to the output power into a conjugate matched load if the antenna is passive.

The active antenna available power in turn leads to generalized definitions for effective area and antenna noise temperature. Effective area retains its traditional definition, but with available power taken to be the active antenna available power. The noise temperature of an antenna is the temperature $T_{\text {iso }}$ of an isotropic noise environment that would be required to produce an isotropic noise response equal to the noise power at the antenna output. As the noise power at the antenna output consists of many contributions, an equivalent temperature can be defined in the same way for each contribution (e.g., external noise, antenna loss noise, or receiver noise). Defined in this way, equivalent temperature might be considered to be "referred to the sky."

Receiving efficiency is the ratio of the isotropic noise response with noiseless antenna (i.e., $P_{\mathrm{t}, \text { iso }}$ less the noise contribution $P_{\text {loss }}$ due to antenna losses) to the isotropic noise response: $\eta_{\text {rec }}=P_{\text {ext,iso }} / P_{\text {t,iso }}=P_{\text {ext,iso }} /\left(P_{\text {ext }, \text { iso }}+P_{\text {loss }}\right)$. If the antenna is passive and reciprocal, the receiving efficiency is equal to the radiation efficiency. With these definitions for receiving efficiency and effective area, antenna efficiency and aperture efficiency can also be defined for active antenna arrays [8].

Finally, the noise matching efficiency $\eta_{\mathrm{n}}$ quantifies the quality of the impedance match between the array element ports and amplifiers included in the receiver system. $\eta_{\mathrm{n}}$ is the ratio of the receiver noise power at the antenna output to the receiver noise power that would result if all amplifiers were ideally noise matched to the element ports. From classical twoport noise theory, the source-referred equivalent temperature of noise added by an amplifier is minimized to $T_{\min }$ when the source reflection coefficient is equal to the amplifier optimal source reflection coefficient noise parameter $\Gamma_{\text {opt }}$. For active antenna arrays, the relevant source reflection coefficients are the active reflection coefficients $\Gamma_{\text {act }}$ looking into each element port [8]. Noise matching efficiency is unity if the amplifier optimal source reflection coefficients are equal to the active reflection coefficient for each array element port. If the impedance match between an element port and its terminating amplifier is poor, then the equivalent receiver noise referred to the source is large in relation to the minimum possible receiver noise and the noise matching efficiency is low. For mutually coupled arrays with nondiagonal S-parameter matrix, active reflection coefficients depend on phase and gain coefficients in the beamforming network, and hence on the beam scan angle. This implies that noise matching efficiency for a coupled array is beam scan angle-dependent and cannot be unity for all steering angles.

All of these figures of merit apply equally well to analog and digitally beamformed arrays. Even MIMO receivers, for which spatial decoding is accomplished by forming linear combinations of array element outputs with coefficients obtained from channel matrix singular vectors, have a well defined receiving efficiency, antenna efficiency, and noise matching efficiency for each singular vector. The new terms therefore significantly expand the realm of applicability of several of the most fundamental parameters used in antenna theory.

\section{ACKNOWLEDGEMENT}

This work was supported in part by the U.S. National Science Foundation under Grant No. 0821780. The authors express appreciation to Michael Francis, Antoine Roederer, Walter Kahn and other members of the IEEE Antenna Definitions Working Group for valuable discussion and improvements to the terms and definitions.

\section{REFERENCES}

[1] P. W. Hannan, "The element-gain paradox for a phased-array antenna," IEEE Trans. Ant.Propag., vol. 12, pp. 423-433, Jul. 1964.

[2] E. Jacobs, "A figure of merit for signal processing reflector antennas," IEEE Trans. Ant. Propag., vol. 33, pp. 100-101, Jan. 1985.

[3] K. F. Warnick and B. D. Jeffs, "Gain and aperture efficiency for a reflector antenna with an array feed," IEEE Antennas and Wireless Propagation Letters, vol. 5, no. 1, pp. 499-502, 2006.

[4] M. V. Ivashina, R. Maaskant, and B. Woestenburg, "Equivalent system representation to model the beam sensitivity of receiving antenna arrays," IEEE Ant. Wireless Propag. Lett., vol. 7, pp. 733-737, 2008.

[5] K. F. Warnick and B. D. Jeffs, "Efficiencies and system temperature for a beamforming array," IEEE Antennas and Wireless Propagation Letters, vol. 7, pp. 565-568, 2008.

[6] K. Warnick, M. Ivashina, R. Maaskant, and B. Woestenburg, "Unified Definitions of Efficiencies and System Noise Temperature for Receiving Antenna Arrays," IEEE Transactions on Antennas and Propagation, vol. 58, no. 6, pp. 2121-2125, 2010.

[7] "IEEE standard definitions of terms for antennas." IEEE Std 145-1993.

[8] K. F. Warnick, B. Woestenburg, L. Belostotski, and P. Russer, "Minimizing the noise penalty due to mutual coupling for a receiving array," IEEE Trans. Ant. Propag., vol. 57, pp. 1634-1644, June 2009. 